\title{
Technique for Direct eV-Scale Measurements of the Mu and Tau Neutrino Masses Using Supernova Neutrinos
}

\author{
J.F. Beacom* \\ Physics Department 161-33, California Institute of Technology, Pasadena, California 91125 \\ R. N. Boyd ${ }^{\dagger}$ \\ Departments of Astronomy and Physics, The Ohio State University, Columbus, Ohio 43210
}

A. Mezzacappa

Physics Division, Oak Ridge National Laboratory, Oak Ridge, Tennessee 37831

(Received 1 June 2000)

\begin{abstract}
Early black hole formation in a core-collapse supernova will abruptly truncate the neutrino fluxes. The sharp cutoff can be used to make model-independent time-of-flight neutrino mass tests. Assuming a neutrino luminosity of $10^{52} \mathrm{erg} / \mathrm{s}$ per flavor at cutoff and a distance of $10 \mathrm{kpc}$, Super-Kamiokande can detect an electron neutrino mass as small as $1.8 \mathrm{eV}$, and the proposed OMNIS detector can detect $\mathrm{mu}$ and tau neutrino masses as small as $6 \mathrm{eV}$. We present the first technique with direct sensitivity to $\mathrm{eV}$-scale mu and tau neutrino masses.
\end{abstract}

PACS numbers: 96.40.Tv, 14.60.Pq, 97.60.Bw, 97.60.Lf

Introduction. - Despite decades of experimental effort, the values of the neutrino masses remain elusive. While the laboratory bound on the electron neutrino mass is about $3 \mathrm{eV}$ [1], the laboratory bounds on the mu and tau neutrino masses are much weaker: $170 \mathrm{keV}$ [2] and $18 \mathrm{MeV}$ [3], respectively. Only recently have neutrino oscillation experiments found strong evidence for nonzero differences of squared neutrino masses. Once discovered, the values of the neutrino masses may provide important clues to physics beyond the standard model. In some scenarios, e.g., with the seesaw mechanism [4], the mu and tau neutrino masses are expected to be much larger than the electron neutrino mass. If they are at the $\mathrm{eV}$ scale or greater, the neutrino masses could also be important cosmologically as a component of the long-sought dark matter. It is therefore crucial to devise direct tests of the mu and tau neutrino masses with sensitivity reaching the $\mathrm{eV}$ scale. While neutrino mass tests based on cosmological considerations may reach the eV scale, they are indirect (no neutrinos are detected) and depend upon the other cosmological parameters being independently known [5].

The best known possibility for directly measuring the $\mathrm{mu}$ and tau neutrino masses is by time-of-flight measurements of supernova neutrinos, comparing the arrival time of the mu and tau neutrinos to that of the electron neutrinos. However, this is complicated by the long intrinsic duration $(\simeq 10 \mathrm{~s})$ of the neutrino signal and the fact that its detailed characteristics are model dependent. Beacom and Vogel have shown that a technique based on the average arrival times $\langle t\rangle$ is model independent and is sensitive to delays as small as $\simeq 0.1 \mathrm{~s}$ [6]. This would allow detection of mu or tau neutrino masses down to $45 \mathrm{eV}$ in SuperKamiokande (SK) and $30 \mathrm{eV}$ in the Sudbury Neutrino Observatory (SNO). If the mu and tau neutrino masses (strictly speaking, those of the relevant mass eigenstates) are nearly degenerate, as suggested by the atmospheric neutrino results [7], then the sensitivity would improve by about $\sqrt{2}$. Unfortunately, it seems difficult to improve the results with this technique, since the mass sensitivity scales with the detector mass $M_{D}$ as $1 / M_{D}^{1 / 4}[6]$. To reach the few-eV scale would require detectors $10^{4}$ times larger, which seems impossible.

In this Letter, we discuss a new time-of-flight technique for measuring neutrino masses that can reach the $\mathrm{eV}$ scale. This technique is applicable if the protoneutron star forms a black hole early enough to abruptly terminate the neutrino signal. We state only our most important results; the details will be discussed at length in a forthcoming paper [8].

Expected neutrino signal. - We consider black hole formation which occurs soon $(\sim 1 \mathrm{~s})$ after core collapse (other scenarios are considered in Ref. [8]). Black hole formation is triggered by accretion, which drives the protoneutron star mass above the maximum stable neutron star mass. The neutrino signal expected in this scenario has been studied by Burrows [9] and Mezzacappa and Bruenn [10]. In these models, the neutrino luminosities were fairly constant at more than $10^{52} \mathrm{erg} / \mathrm{s}$ per flavor until abruptly terminated by black hole formation. In fact, the transition should have a nonzero duration, of order the light crossing time $2 R / c \simeq 0.1 \mathrm{~ms}$, as the protoneutron star radius shrinks to that of the final black hole. During the transition, the gravitational redshift, originally $\simeq 10 \%$, rapidly diverges, truncating the neutrino signal. Using a singularityavoiding code, Baumgarte et al. [11] studied the transition and found its duration to be $0.5 \mathrm{~ms}$. Thus, we can consider the neutrino fluxes to be sharply and simultaneously terminated.

The results below assume a luminosity $L_{\mathrm{BH}}=$ $10^{52} \mathrm{erg} / \mathrm{s}$ per flavor at the cutoff time $t_{\mathrm{BH}}$, and a distance $D=10 \mathrm{kpc}$. We assume the following temperatures: 
$T=3.5 \mathrm{MeV}$ for $\nu_{e}, T=5 \mathrm{MeV}$ for $\bar{\nu}_{e}$, and $T=$ $8 \mathrm{MeV}$ for $\nu_{\mu}, \nu_{\tau}, \bar{\nu}_{\mu}$, and $\bar{\nu}_{\tau}$. It will be shown that the necessary quantities can be measured in a realistic situation.

Neutrino mass effects. - At lowest order, a neutrino with mass $m$ (in $\mathrm{eV}$ ) and energy $E$ (in $\mathrm{MeV}$ ) will have an energy-dependent delay (in s) relative to a massless neutrino in traveling over a distance $D$ (in $10 \mathrm{kpc}$ ):

$$
\Delta t(E)=0.515\left(\frac{m}{E}\right)^{2} D
$$

The distance is scaled by the approximate distance to the Galactic center, though a supernova may be detected from anywhere in the Galaxy. For the smallest detectable masses, the delay effects will be visible only after the sharp cutoff, where no events are otherwise expected. Since the delays are very small, the luminosities and temperatures can be taken as constant over the short interval before $t_{\mathrm{BH}}$. The event rate for $t>t_{\mathrm{BH}}$ is [8]

$$
\frac{d N}{d t}(t)=C\left[\frac{L_{\mathrm{BH}}}{10^{51} \mathrm{erg} / \mathrm{s}}\right] \int_{0}^{E_{\max }} d E f(E)\left[\frac{\sigma(E)}{10^{-42} \mathrm{~cm}^{2}}\right],
$$

where $f(E)$ is the neutrino energy spectrum and $\sigma(E)$ the cross section. The upper limit $E_{\max }$ on the integral allows only delays as large as $t-t_{\mathrm{BH}}$, i.e.,

$$
E_{\max }=m \sqrt{\frac{0.515 D}{t-t_{\mathrm{BH}}}},
$$

where the units are as in Eq. (1). Note that the time and neutrino mass dependence appear only through $E_{\max }$. For $t<t_{\mathrm{BH}}, E_{\max } \rightarrow \infty$, and the rate is constant. If the neutrino energy can be measured, as for some charged-current reactions, then the event rates for different neutrino energies can easily be obtained. For an $\mathrm{H}_{2} \mathrm{O}$ detector, the constant $C$ is

$$
C_{\mathrm{H} 2 \mathrm{O}}=(1.74 / \mathrm{s})\left[\frac{M_{D}}{1 \mathrm{kton}}\right]\left[\frac{10 \mathrm{kpc}}{D}\right]^{2}\left[\frac{1 \mathrm{MeV}}{\langle E\rangle}\right] .
$$

For a Fermi-Dirac spectrum, $\langle E\rangle=3.15 T$. The constant for a ${ }^{208} \mathrm{~Pb}$ detector can be obtained by scaling by the relative number of targets/kton, i.e., 18/208.

The expected number of delayed counts after $t_{\mathrm{BH}}$ can be calculated using Eq. (2). This will be useful when $t_{\mathrm{BH}}$ can be measured independently. It can be shown [8] that this has the very simple form:

$$
N_{\mathrm{del}}=\frac{d N}{d t}\left(t_{\mathrm{BH}}\right) \times 0.515\left(\frac{m}{E_{c}}\right)^{2} D,
$$

where the event rate is in $\mathrm{s}^{-1}$, and the other units are as in Eq. (1). This formula would obviously be true if only a single energy contributed and the sharp cutoff in the event rate were rigidly translated by the delay. But it is remarkable and very convenient that it is still true even when there is a spectrum of energies and the event rate

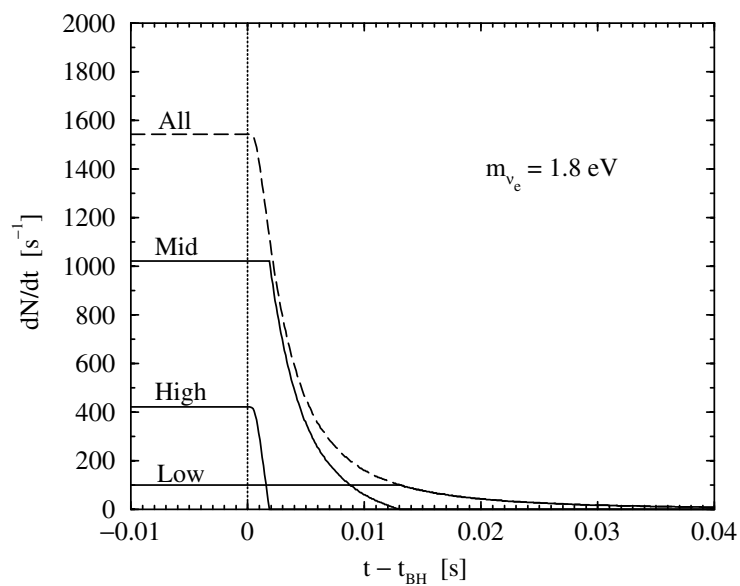

FIG. 1. The event rates due to $\bar{\nu}_{e}+p \rightarrow e^{+}+n$ in SK, for different ranges of the neutrino energy: "Low" $(0 \leq$ $E \leq 11.3 \mathrm{MeV}$, contains 2.4 events past the true $t_{\mathrm{BH}}$ ), "Mid" (11.3 $\leq E \leq 30 \mathrm{MeV}, 4.8$ events), "High" (30 $\leq E \leq \infty \mathrm{MeV}$, 0.5 events), and "All" (all energies, 7.7 events). Note that only the rate after about $t_{\mathrm{BH}}$ is shown, and that the range of $t-t_{\mathrm{BH}}$ is very short.

develops a decaying tail past the cutoff (as in Figs. 1 and 2 ). The physical significance of the "central" energy $E_{c}$ is that it is (to an excellent approximation) simply the Gamow peak of the falling thermal spectrum and the rising cross section. As derived, this is an exact result.

Electron neutrino mass. - We first consider the measurement of $t_{\mathrm{BH}}$ and $m_{\nu_{e}}$ using the $\bar{\nu}_{e}+p \rightarrow e^{+}+n$ events in the 32-kton SK detector. For $T=5 \mathrm{MeV}$, the thermally averaged cross section (for the sum of the two protons in $\mathrm{H}_{2} \mathrm{O}$ ) is $44 \times 10^{-42} \mathrm{~cm}^{2}$ [12]. The event rate at or before $t_{\mathrm{BH}}$ is thus $\simeq 1500 \mathrm{~s}^{-1}$. After $t_{\mathrm{BH}}$, the rate is zero if $m_{\nu_{e}}=0$ and will develop a tail if $m_{\nu_{e}}>0$.

For a sharp edge, the edge position can be determined with an error given by the reciprocal of the event rate before the edge, i.e., the event spacing [8,13]. If we knew that $m_{\nu_{e}}=0$, then $t_{\mathrm{BH}}$ would be determined to $\simeq 1 \mathrm{~ms}$. More realistically, a mass as large as the laboratory bound, $m_{\nu_{e}} \lesssim 3 \mathrm{eV}$ [1], would cause delays as large as $40 \mathrm{~ms}$, so that the extracted $t_{\mathrm{BH}}$ would be too large.

However, we can simultaneously measure $m_{\nu_{e}}$ and $t_{\mathrm{BH}}$ by splitting the $\bar{\nu}_{e}+p \rightarrow e^{+}+n$ data into different ranges of neutrino energy (using $E_{\nu} \simeq E_{e}+1.3 \mathrm{MeV}$ ). These are defined in the caption of Fig. 1. The Low group must be excluded from consideration because these events have positron total energy less than $10 \mathrm{MeV}$, and can be confused with the 5-10 MeV gammas from neutral-current reactions on ${ }^{16} \mathrm{O}$ [14]. The High group has very little delay and will thus primarily be sensitive to $t_{\mathrm{BH}}$. Then the Mid group will determine $m_{\nu_{e}}$, by counting events delayed past the $t_{\mathrm{BH}}$ determined by the High group.

In Fig. 1, we show a possible analysis for the case of $m_{\nu_{e}}=1.8 \mathrm{eV}$. In the High group, the number of events in the tail is $\lesssim 1$, so the cutoff appears sharp and is specified to within $\simeq 2 \mathrm{~ms}$. This uncertainty affects the expected 


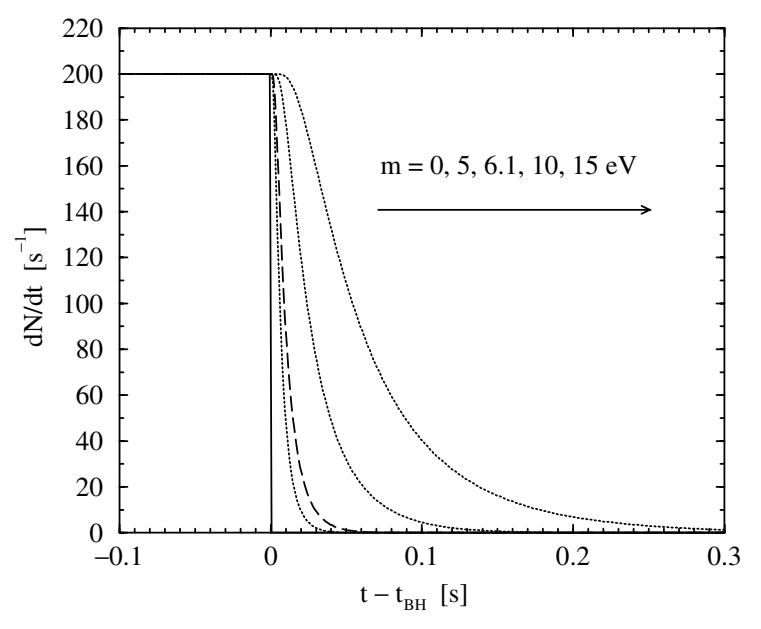

FIG. 2. The results for the combined 1-n neutral-current event rate due to $\nu_{\mu}, \nu_{\tau}, \bar{\nu}_{\mu}$, and $\bar{\nu}_{\tau}$ in OMNIS. Note that only the rate after about $t_{\mathrm{BH}}$ is shown. Before $t_{\mathrm{BH}}$, other reactions contribute about $20 \%$ of the total neutron rate; they are not included here, and will have to be statistically subtracted from the measured rate. The mu and tau neutrino masses are assumed degenerate [7]. The $m=0$ case is drawn with a solid line. The $m=6.1 \mathrm{eV}$ case, with 2.3 events expected in the tail, is the first case that can be reliably distinguishable from $m=0$, and is drawn with a long-dashed line. The results for other masses are drawn with dotted lines.

number in the Mid group by $\simeq 2$ events. Even so, one can still reliably see a few delayed counts after the measured $t_{\mathrm{BH}}$, enough to establish a nonzero mass (the statistics are discussed in more detail below). A more sophisticated fit would improve our results somewhat, and we assume a final uncertainty on $t_{\mathrm{BH}}$ of about $1 \mathrm{~ms}$. For a supernova in which the neutrino fluxes are not truncated by black hole formation, SK could detect an electron neutrino mass as small as $\sim 3 \mathrm{eV}[15]$.

Mu and tau neutrino masses. - We consider mu and tau neutrino detection in OMNIS, a proposed supernova neutrino detector based on lead and iron [16]. (Certain practical effects make the neutrino mass sensitivity of SK and SNO somewhat worse than OMNIS [8]). Since their energies are below the charged-current thresholds, supernova $\mathrm{mu}$ and tau neutrinos can be detected only by their neutralcurrent interactions. On the other hand, because of the temperature hierarchy, they will dominate the neutral-current yields. In OMNIS, the dominant neutral-current reaction is the spallation of single neutrons from lead. The neutrons could be detected by capture in a gadolinium-doped liquid scintillator, which yields an $8-\mathrm{MeV}$ gamma cascade in about $0.030 \mathrm{~ms}$ (much smaller than typical mass delays).

For $T=8 \mathrm{MeV}$, the thermally averaged cross section for the sum of $\nu_{\mu}$ and $\bar{\nu}_{\mu}$ (or $\nu_{\tau}$ and $\bar{\nu}_{\tau}$ ) on ${ }^{208} \mathrm{~Pb}$, including the one-neutron spallation probability, is $760 \times$ $10^{-42} \mathrm{~cm}^{2}$ [17]. The cross sections on ${ }^{206} \mathrm{~Pb}$ and ${ }^{207} \mathrm{~Pb}$, which together comprise $46 \%$ of natural lead, are expected to be similar [8]. For a supernova at $10 \mathrm{kpc}$ in which the neutrino fluxes are not cut off by black hole formation, we

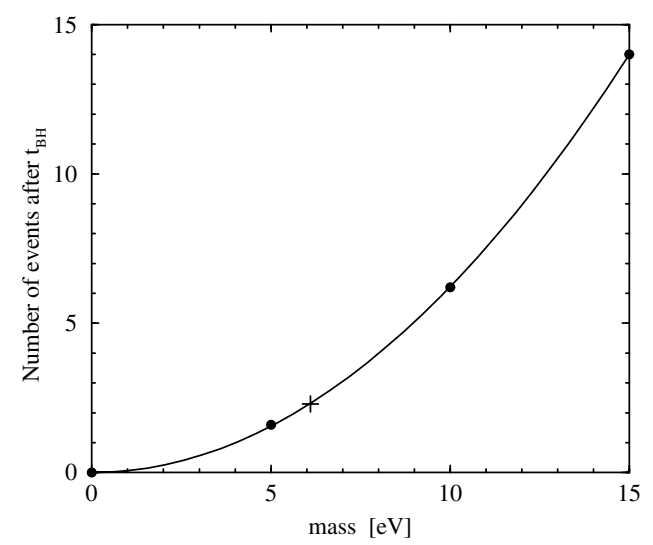

FIG. 3. The expected number of delayed counts $N_{\text {del }}$ in OMNIS as a function of the neutrino mass. The points are obtained by direct numerical integration of Eq. (2). The "+" indicates the smallest discernible mass at the $90 \%$ C. L. The solid line is obtained with Eq. (5), using $E_{c}=40.7 \mathrm{MeV}$, the Gamow peak energy.

assume that OMNIS will have $\simeq 1000$ one-neutron neutralcurrent events due to $\nu_{\mu}, \nu_{\tau}, \bar{\nu}_{\mu}$, and $\bar{\nu}_{\tau}$ on lead (the events on iron are not included in our calculations). This goal could be met with a 2.2 kton lead detector with perfect neutron detection efficiency. A realistic design based on 4 kton of lead and 10 kton of iron, and with about this many events, is described by Boyd [16].

In Fig. 2, we plot the relevant neutral-current rate for different values of the neutrino mass, calculated using Eq. (2). In Fig. 3, we plot the number of delayed events $N_{\text {del }}$ as a function of the neutrino mass, using Eq. (5) and by direct integration. Equation (5) is remarkable for its simplicity, and also because it is written in terms of measurable quantities. The cutoff time $t_{\mathrm{BH}}$ will be measured in SK. The neutral-current event rate at or before $t_{\mathrm{BH}}$ will be measured in OMNIS, as will $N_{\text {del }}$. The central energy $E_{c}$ depends on the mu and tau neutrino temperature, which can be estimated by the neutral-current yields on different targets [8]. We assume that the distance $D$ can be determined by astronomical means.

Given the measured value of $N_{\text {del }}$, Eq. (5) can be immediately solved for the best-fit neutrino mass. If $N_{\text {del }}=0$ is measured, then the best-fit mass is $m=0$, and an upper limit can be placed. An expectation of 2.3 counts fluctuates down to 0 counts only $10 \%$ of the time. Thus, setting $N_{\text {del }}=2.3$, an upper limit on the mass $m_{\text {lim }}$ is obtained. This is the largest mass, given the expected Poisson statistics, that could be confused with the massless case. For the present case, this is $6.1 \mathrm{eV}$.

Since the fractional error on $N_{\text {del }}$ due to Poisson statistics is large $(\simeq 1 / \sqrt{2.3} \simeq 65 \%)$, errors on other inputs are expected to be irrelevant. The uncertainty on $t_{\mathrm{BH}}$ from SK is assumed to be about $1 \mathrm{~ms}$. From Fig. 2, this uncertainty can be seen to change the expected number $N_{\text {del }}$ by $\simeq 0.2$ events, which is negligible. Other possible errors, e.g., the detector background, the disregarded $0.5 \mathrm{~ms}$ tail 
of the luminosity, and $\nu_{e}$ and $\bar{\nu}_{e}$ events after $t_{\mathrm{BH}}$, are even less important [8].

For a supernova that does not have the sharp cutoff in the rate characteristic of black hole formation, the modelindependent $\langle t\rangle$ analysis [6] yields an $m_{\mathrm{lim}}$ that is independent of the distance $D$ and scales as $1 / M_{D}^{1 / 4}$ [6]. For the present case, $m_{\text {lim }}$ scales as

$$
m_{\lim } \sim E_{c} \sqrt{\frac{\langle E\rangle D}{\sigma_{\mathrm{eff}} L_{\mathrm{BH}} M_{D}}},
$$

where $\sigma_{\text {eff }}$ is the thermally averaged cross section. In terms of absolute sensitivity, these techniques compare as 21 and $6 \mathrm{eV}$, respectively. These differences are consequences of the sharp cutoff in the neutrino flux.

Conclusions. - If a black hole forms early in a corecollapse supernova, then the fluxes of the various flavors of neutrinos will be abruptly and simultaneously terminated when the neutrinospheres are enveloped by the event horizon. For a massive neutrino, the cutoff in the arrival time will be delayed by $\Delta t \sim(m / E)^{2}$ relative to a massless neutrino.

The Galactic core-collapse supernova rate is about 3 per century or higher [8], and the work of Brown and Bethe [18] suggests that black holes are formed about half of the time. In the work of Burrows [9] and Mezzacappa and Bruenn [10], the neutrino luminosities just before black hole formation are very high. These results indicate that there is a reasonably good chance that such an event could be observed by the present and proposed supernova neutrino detectors [8]. If so, there are important practical consequences.

First, since SK can measure the neutrino energy of the $\bar{\nu}_{e}+p \rightarrow e^{+}+n$ events, both $t_{\mathrm{BH}}$ and $m_{\nu_{e}}$ can be measured by the arrival times for different neutrino energies. An electron neutrino mass as small as $1.8 \mathrm{eV}$ can be detected. Second, although the mu and tau neutrino energies are not measured in their neutral-current detection reactions, their masses can be measured by counting the number of events after $t_{\mathrm{BH}}$. In the proposed OMNIS detector, a mu and tau neutrino mass (assumed degenerate [7]) as small as $6 \mathrm{eV}$ can be detected. This is the only known direct technique with $\mathrm{eV}$-scale sensitivity for these masses. Third, these results scale with the distance, luminosity, and detector mass as $\sqrt{D / L_{\mathrm{BH}} M_{D}}$. This favorable scaling with the detector mass suggests that it would be realistic to consider even larger detectors, in order to reach 1 or $2 \mathrm{eV}$ for all three neutrino masses.

J.F. B. was supported by Caltech. R. N.B. was supported by NSF Grant No. PHY-9901241. A. M. is supported at the Oak Ridge National Laboratory, managed by UT-Battelle, LLC, for the U.S. Dept. of Energy under Contract No. DE-AC05-00OR22725. We thank Felix Boehm, Steve Bruenn, Will Farr, Josh Grindlay, Manoj Kaplinghat, Gail McLaughlin, Alex Murphy, Yong-Zhong Qian, Petr Vogel, and Jerry Wasserburg for discussions.

*Present address: Theoretical Astrophysics, MS-209, Fermilab, P.O. Box 500, Batavia, IL 60510.

Electronic address: beacom@fnal.gov

†Electronic address: boyd@mps.ohio-state.edu

\#lectronic address: mezz@nova.phy.ornl.gov

[1] Ch. Weinheimer et al., Phys. Lett. B 460, 219 (1999); V. M. Lobashev et al., Phys. Lett. B 460, 227 (1999).

[2] K. Assamagan et al., Phys. Rev. D 53, 6065 (1996).

[3] R. Barate et al., Eur. Phys. J. C 2, 395 (1998).

[4] M. Gell-Mann, P. Ramond, and R. Slansky, in Supergravity, edited by P. van Nieuwenhuizen and D. Z. Freedman (North-Holland, Amsterdam, 1979).

[5] C. P. Ma, Nucl. Phys. Proc. Suppl. 51B, 254 (1996); W. Hu, D. J. Eisenstein, and M. Tegmark, Phys. Rev. Lett. 80, 5255 (1998); M. Kaplinghat, R. E. Lopez, S. Dodelson, and R. J. Scherrer, Phys. Rev. D 60, 123508 (1999); A. R. Cooray, Astron. Astrophys. 348, 31 (1999); R. A. Croft, W. Hu, and R. Dave, Phys. Rev. Lett. 83, 1092 (1999); M. Fukugita, G. Liu, and N. Sugiyama, Phys. Rev. Lett. 84, 1082 (2000); E. Gawiser, astro-ph/0005365.

[6] J. F. Beacom and P. Vogel, Phys. Rev. D 58, 053010 (1998); 58, 093012 (1998).

[7] Y. Fukuda et al., Phys. Rev. Lett. 81, 1562 (1998).

[8] J.F. Beacom, R. N. Boyd, and A. Mezzacappa (to be published).

[9] A. Burrows, Astrophys. J. 334, 891 (1988).

[10] A. Mezzacappa and S. W. Bruenn, in The Identification of Dark Matter, edited by N. J. C. Spooner and V. Kudryavtsev (World Scientific, Singapore, 1999).

[11] T. W. Baumgarte, S.A. Teukolsky, S. L. Shapiro, H. T. Janka, and W. Keil, Astrophys. J. 468, 823 (1996).

[12] P. Vogel and J. F. Beacom, Phys. Rev. D 60, 053003 (1999).

[13] R. V. Hogg and A. T. Craig, Introduction to Mathematical Statistics (Macmillan, New York, 1978), 4th ed.

[14] K. Langanke, P. Vogel, and E. Kolbe, Phys. Rev. Lett. 76, 2629 (1996).

[15] T. Totani, Phys. Rev. Lett. 80, 2039 (1998).

[16] R. N. Boyd, in Origins of Matter and Evolution of Galaxies, edited by T. Kajino and S. Kubono (World Scientific, Singapore, 2000).

[17] G. M. Fuller, W. C. Haxton, and G. C. McLaughlin, Phys. Rev. D 59, 085005 (1999).

[18] G.E. Brown and H.A. Bethe, Astrophys. J. 423, 659 (1998); H. A. Bethe and G.E. Brown, Astrophys. J. 445, L129 (1995). 